\title{
Interference Constrained Device-to-Device Communications
}

\author{
Serveh Shalmashi*, Guowang Miao*, Zhu Han ${ }^{\dagger}$, and Slimane Ben Slimane* \\ *Dept. of Communication Systems, School of ICT, KTH Royal Institute of Technology, Stockholm, Sweden \\ ${ }^{\dagger}$ Dept. of Electrical and Computer Engineering, University of Houston, Houston, TX, USA \\ E-mail:\{serveh,guowang,slimane\}@kth.se, and zhan2@uh.edu
}

\begin{abstract}
This paper considers a scenario in which multiple device-to-device (D2D) users can reuse the uplink resources of a cellular network to transmit directly to their corresponding receivers. The aggregated interference from the D2D users is limited by applying a threshold on the allowable interference in the base station. The problem is solved under two types of constraints, namely, the peak interference and average interference constraints. In the former, we assume that full channel state information (CSI) is available at the base station, and we optimize the allowable transmit power for the D2D users so that the number of coexisting D2D communications is maximized. We further define a quality-of-service constraint for the D2D users. In practice, however, it is difficult to have complete CSI at the base station as it imposes heavy signaling overhead. Therefore, in the latter scenario, we assume that no knowledge about the location of D2D users and their CSI are available at the base station. This approach does not impose any signaling overhead. Our results show that even with no CSI knowledge, we are able to improve the system performance in terms of throughput by allowing coexisting D2D communications while satisfying the cellular user's constraints.
\end{abstract}

\section{INTRODUCTION AND RELATED WORK}

Spectrum sharing is one way of reusing spectrum in both temporal and spatial domains and has been frequently used to improve the spectral efficiency of wireless networks. Deviceto-device (D2D) communication has recently been proposed as an underlay for cellular networks to improve the spectrum reuse. In the D2D mode, transmission is done using a direct link from one device to another device as opposed to the traditional cellular mode in which all transmissions go through base stations (BS) [1]-[3].

D2D communications provide potential gains for cellular networks. First, since D2D users reuse resources of cellular users, the network can achieve a reuse factor less than one. This potentially leads to an increase in network capacity [4] as well as a decrease in the load of base stations. In addition, proximity of users results in power saving for both base stations and mobile terminals. Higher data rates can also be achieved due to using just one hop in D2D mode. Moreover, D2D communication may extend cell coverage [3]. However, since both cellular and D2D users employ the same set of network resources, their achievable gains depend on the level of interference from and to the D2D users. Therefore, interference management, mode selection, and efficient

Part of this work has been performed in the framework of the FP7 project ICT-317669 METIS. resource scheduling are important for implementation of D2D communications [5]. In order to manage the interference of D2D links to the cellular network, one approach is to limit the maximum transmit power of the D2D users as considered in [6] and [7]. Another approach is minimizing the received interference while maximizing the number of D2D links in the cell [8]. Proper pairing for maximizing the network capacity while minimizing the interference is considered in [9].

D2D communications can reuse resources in the downlink, the uplink, or both. This paper will focus on reusing uplink resources because reusing downlink resource may lead to regulatory issues [10]. Our objective is to maximize the number of D2D users that can simultaneously share the resources of one cellular user so that the spectrum reuse is optimized. The problem is formulated under two different constraints for the cellular users. First, we investigate this problem under the peak interference constraint where instantaneous channel state information (CSI) is available. The optimal number of active D2D users is found for this problem. Next, we formulate the problem under the average interference constraint where no knowledge of the location of D2D users and their CSI is available. We derive an upper bound on the number of D2D users that can transmit simultaneously with no CSI knowledge. The immediate benefit of such a solution is that the BS can estimate the allowable number of coexisting D2D communications without requiring any CSI, and therefore, if any new D2D pair is discovered, the BS can determine if this user can be scheduled in the resource of the cellular user. Furthermore, we also consider quality-of-service (QoS) requirements for D2D users.

In our previous work [11], we have investigated the tradeoff between CSI signaling overhead and overall system performance in terms of the cell sum throughput for a similar scenario, but under a more restrictive assumption that the received interference power in the BS from all active D2D users are equal. In this work, we will find the optimal solution for the problem of admission and power control for D2D users with QoS requirements under the peak interference constraint. Moreover, in our second scenario under the average interference constraint and no CSI, an upper bound on the number of D2D users that can be scheduled is derived.

The rest of this paper is organized as follows: Section II presents our system model. In Section III, the problem of power and admission control for D2D users under the peak 


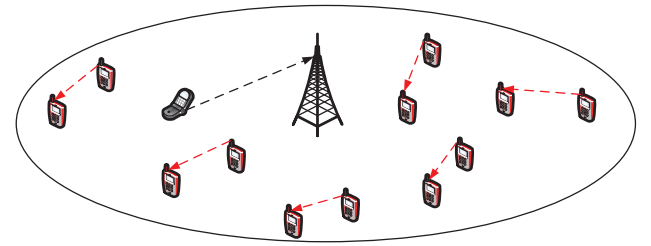

Fig. 1. System model with one cellular and multiple D2D users.

interference constraint is formulated, and in Section IV the problem is analyzed under the average interference constraint. The performance of the system under both constraints are evaluated in Section V. Finally, the paper is concluded in Section VI.

\section{SYSTEM MODEL}

We consider a single-cell cellular network with one cellular user equipment (CUE) communicating with the BS as depicted in Fig. 1. In addition, there are $K$ other users in the cell that can communicate directly with their corresponding receivers in the D2D mode. We assume that the D2D communications use uplink resources of the network. Both cellular and D2D users are distributed uniformly over the cell area.

When a user is operating in the D2D mode, it reuses the resources of the network and causes interference at the BS for the CUE transmission over the same channel in the uplink. We assume more than one D2D user can transmit simultaneously. Therefore, the aggregated interference from the active D2D users at the $\mathrm{BS}$ is an important issue. Besides, the interference caused by the cellular user to the $\mathrm{D} 2 \mathrm{D}$ resources degrades the performance of D2D communications. Thus, D2D users that cannot fulfill the QoS requirements should not use the D2D mode.

The goal is to maximize the number of active D2D users in the cell while ensuring that the aggregated interferences to both cellular and D2D users meet their QoS requirements. To model the active D2D users, we define a binary random variable $x_{k} \in\{0,1\}, k \in\{1, \ldots, K\}$, where $x_{k}=1$ corresponds to the event that the $k$ th $\mathrm{D} 2 \mathrm{D}$ user is active; $x_{k}=0$, otherwise. Let $G_{i j}$ denote the instantaneous channel gain from the $i$ th transmitter to the $j$ th receiver with $i, j \in\{0,1, \ldots, K\}$, where $i, j=0$ corresponds to the CUE and the BS, and the D2D users otherwise. The instantaneous channel gain accounts for the path loss, $\bar{g}_{i j}$, and Rayleigh fading, $\left|h_{i j}\right|^{2}$, with $\mathbb{E}\left[\left|h_{i j}\right|^{2}\right]=1$, i.e.,

$$
G_{i j}=\bar{g}_{i j}\left|h_{i j}\right|^{2} \text {. }
$$

Define the cellular user's and the $k$ th D2D user's signal-tonoise-plus-interference ratio (SINR), respectively, as

$$
\begin{aligned}
\gamma_{0} & =\frac{p_{0} G_{00}}{\sum_{k=1}^{K} x_{k} p_{k} G_{k 0}+\sigma_{0}^{2}}, \\
\gamma_{k} & =\frac{x_{k} p_{k} G_{k k}}{\sum_{k^{\prime} \neq k}^{K} x_{k^{\prime}} p_{k^{\prime}} G_{k^{\prime} k}+p_{0} G_{0 k}+\sigma_{k}^{2}},
\end{aligned}
$$

where $p_{j}, j \in\{0,1, \ldots, K\}$, are the transmit powers, and $\sigma_{j}^{2}$ is the receiver noise power.

\section{Peak Interference Constraint}

We assume that the CUE transmits with power $p_{0}$. The SINR constraint for the cellular user is written as

$$
\gamma_{0} \geq \gamma_{0}^{\text {th }},
$$

where $\gamma_{0}^{\text {th }}$ is a pre-determined threshold for the CUE's instantaneous SINR. The SINR constraint in (4) can equivalently be written as the aggregated peak interference constraint,

$$
\sum_{k=1}^{K} x_{k} p_{k} G_{k 0} \leq I_{\mathrm{th}}
$$

with $I_{\text {th }}=p_{0} G_{00} / \gamma_{0}^{\text {th }}-\sigma_{k}^{2}$. This constraint protects the cellular receiver from the active D2D users' interference.

Users have a choice to be active in either the D2D mode or conventional cellular mode. They often require certain QoS, which can be written in terms of individual SINR constraints as

$$
\frac{x_{k} p_{k} G_{k k}+\left(1-x_{k}\right) M_{k}}{\sum_{j=1, j \neq k}^{K} x_{j} p_{j} G_{j k}+p_{0} G_{0 k}+\sigma_{k}^{2}} \geq \gamma_{d}^{\text {th }}, \quad \forall k \in\{1, \ldots, K\} .
$$

The term $\left(1-x_{k}\right) M_{k}$ in the numerator is to overcome the issue of infeasibility of the optimization problem because of inactive D2D users. That is, we choose the coefficient $M_{k}$ in such a way that when user $k$ is not scheduled, i.e., $x_{k}=0$, the corresponding SINR constraint is satisfied and does not make the problem infeasible. Therefore, we have [12]

$$
M_{k} \geq \gamma_{d}^{\text {th }}\left(P_{\max }^{d} \sum_{j=1, j \neq k}^{K} G_{j k}+P_{\max }^{c} G_{0 k}+\sigma_{k}^{2}\right),
$$

where $P_{\max }^{c}$ and $P_{\max }^{d}$ are the maximum transmit powers of the cellular and D2D users, respectively.

The final constraints are to limit the maximum transmit power of the active D2D users, i.e.,

$$
x_{k} p_{k} \leq P_{\max }^{d}, \quad \forall k \in\{1, \ldots, K\} .
$$

Our objective is to maximize the number of active D2D users. In the above constraints, the multiplication of the optimization variables, i.e., the term $x_{k} p_{k}$, makes these constraints nonlinear. To overcome this issue, we define a new variable $p_{x_{k}}=x_{k} p_{k}$. Since $x_{k} \in\{0,1\}$, the variable $p_{x_{k}}$ should also satisfy $p_{x_{k}} \leq P_{\max }^{d}$. Therefore, the new definition does not change any of the constraints except for removing their nonlinearity. We can formulate our optimization problem as

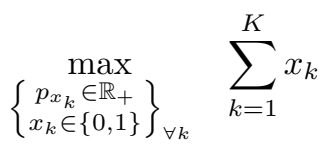

subject to

$\frac{p_{x_{k}} G_{k k}+\left(1-x_{k}\right) M_{k}}{\sum_{j=1, j \neq k}^{K} p_{x_{j}} G_{j k}+p_{0} G_{0 k}+\sigma_{k}^{2}} \geq \gamma_{d}^{\text {th }}, \quad \forall k \in\{1, \ldots, K\}$, 


$$
\begin{array}{ll}
\sum_{k=1}^{K} p_{x_{k}} G_{k 0} \leq I_{\mathrm{th}}, & \\
p_{x_{k}}-P_{\max }^{d} \leq 0, & \forall k \in\{1, \ldots, K\} .
\end{array}
$$

Note that this approach requires complete CSI of all users. The problem (9)-(10) is a mixed integer programming (MIP) problem. However, the constraint (10a) can be rewritten as a linear constraint. Defining $A_{j k}$ as

$$
A_{j k} \triangleq\left\{\begin{array}{cl}
-G_{k k} & \text { if } j=k, \\
\gamma_{d}^{\text {th }} G_{j k} & \text { if } j \neq k,
\end{array}\right.
$$

and $B_{k}$ as

$$
B_{k} \triangleq M_{k}-\gamma_{d}^{\mathrm{th}} p_{0} G_{0 k}-\gamma_{d}^{\mathrm{th}} \sigma_{k}^{2},
$$

the above optimization problem can be recast as a mixed integer linear programming (MILP) problem:

$$
\max _{\left\{\begin{array}{c}
p_{x_{k}} \in \mathbb{R}_{+} \\
x_{k} \in\{0,1\}
\end{array}\right\}_{\forall k}} \sum_{k=1}^{K} x_{k}
$$

subject to

$$
\begin{array}{ll}
\sum_{j=1}^{K} p_{x_{j}} A_{j k}+M_{k} x_{k} \leq B_{k}, & \forall k \in\{1, \ldots, K\}, \\
\sum_{k=1}^{K} p_{x_{k}} G_{k 0} \leq I_{\mathrm{th}}, & \\
p_{x_{k}}-P_{\max }^{d} \leq 0, & \forall k \in\{1, \ldots, K\} .
\end{array}
$$

The solution to the above MILP problem can be found using optimization solvers, and can also be verified by a greedy enumeration approach.

\section{AVERAGE InTERFEREnCE CONSTRAint}

So far, we have assumed that the positions and instantaneous CSI for all D2D users are known, based on which we could determine the optimal number of active D2D users with respect to their QoS as well as the CUE's. However, having all this information at the BS requires heavy signaling and power consumption in the transmitters, and may not always be possible due to delay constraints. Therefore, the question is if it is possible to estimate the number of D2D users that can be active and simultaneously share the spectrum with one CUE without the knowledge of users' positions and their CSI.

Since the location of D2D users are unknown to the BS, the distance between a D2D transmitter to the BS as well as its corresponding receiver is a random variable. Therefore, the distance-dependent path loss is not a deterministic function anymore and only statistics of the path loss model are desirable. The transmit power of the D2D user is also affected by this assumption and becomes a random variable as well, since we use power control in order to assure the link quality and to manage the interference in the shared environment. There may be different power control policies [13]. We consider a classic power control policy, the channel inversion, which assumes constant received power in the receiver in order for the signal to be reliably detected. Using this policy, the transmit power $p_{k}$ of the $k$ th D2D user is

$$
p_{k}=\frac{p_{r_{k}}}{G_{k k}}
$$

where $p_{r_{k}}=\Gamma_{k} \sigma_{k}^{2}$ is the received power at the $k$ th D2D receiver and $\Gamma_{k}$ is its desired SNR based on the required data rate. The value of $p_{r_{k}}$ is channel-independent. Assuming all D2D users have the same rate requirement and the same receiver noise, i.e., $\Gamma_{k}=\gamma_{d}^{\text {th }}$ and $\sigma_{k}^{2}=\sigma^{2}$, we have

$$
p_{r_{k}}=\gamma_{d}^{\text {th }} \sigma^{2} \triangleq p_{r}, \quad k \in\{1, \ldots, K\} .
$$

The transmit power $p_{k}$ in (15) depends on the channel between the D2D transmitter and its own receiver, and is independent of the interference channels.

We use average interference constraint as the QoS constraint for the CUE. That is, we change the constraint (10b) to

$$
\mathbb{E}_{p, G}\left[\sum_{k=1}^{K} x_{k} p_{k} G_{k 0}\right] \leq I_{\mathrm{th}} .
$$

Using channel inversion as the power control policy, we have

$$
\mathbb{E}_{G}\left[\sum_{k=1}^{K} x_{k} p_{r} \frac{G_{k 0}}{G_{k k}}\right] \leq I_{\mathrm{th}} .
$$

Since the ratio $\frac{G_{k 0}}{G_{k k}}$ is a random variable, the left hand side of (18) is a weighted sum of, and therefore, a linear function in the random variables. Thus, by the Jensen's inequality for convex functions [14], we can write

$$
\sum_{k=1}^{K} x_{k} p_{r} \mathbb{E}_{G}\left[\frac{G_{k 0}}{G_{k k}}\right] \leq I_{\mathrm{th}} .
$$

In (1), the path loss between a user and the BS is modeled as $\bar{g}_{k 0}=c_{0} d_{k 0}^{-\alpha_{0}}$ where $c_{0}$ and $\alpha_{0}$ are known path loss coefficient and exponent, respectively. The path loss between a D2D transmitter and its receiver is modeled similarly with $c_{d}$ and $\alpha_{d}$. Then, we can lower bound the left hand side of (19) as

$$
\begin{aligned}
& \sum_{k=1}^{K} x_{k} p_{r} \mathbb{E}_{G}\left[\frac{G_{k 0}}{G_{k k}}\right] \stackrel{(a)}{=} p_{r} \sum_{k=1}^{K} x_{k} \mathbb{E}_{G}\left[G_{k 0}\right] \mathbb{E}_{G}\left[\frac{1}{G_{k k}}\right] \\
& =p_{r} \sum_{k=1}^{K} x_{k} \mathbb{E}_{G}\left[\bar{g}_{k 0}\left|h_{k 0}\right|^{2}\right] \mathbb{E}_{G}\left[\frac{1}{\bar{g}_{k k}\left|h_{k k}\right|^{2}}\right] \\
& \stackrel{(b)}{=} \frac{p_{r} c_{0}}{c_{d}} \sum_{k=1}^{K} x_{k} \mathbb{E}_{d}\left[d_{k 0}^{-\alpha_{0}}\right] \mathbb{E}_{h}\left[\left|h_{k 0}\right|^{2}\right] \mathbb{E}_{d}\left[\frac{1}{d_{k k}^{-\alpha_{d}}}\right] \mathbb{E}_{h}\left[\frac{1}{\left|h_{k k}\right|^{2}}\right] \\
& \stackrel{(c)}{\geq} \frac{p_{r} c_{0}}{c_{d}} \sum_{k=1}^{K} x_{k} \mathbb{E}_{d}\left[d_{k 0}^{-\alpha_{0}}\right] \mathbb{E}_{h}\left[\left|h_{k 0}\right|^{2}\right] \mathbb{E}_{d}\left[d_{k k}^{\alpha_{d}}\right] \frac{1}{\mathbb{E}_{h}\left[\left|h_{k k}\right|^{2}\right]} \\
& \stackrel{(d)}{=} \frac{p_{r} c_{0}}{c_{d}} \sum_{k=1}^{K} x_{k} \mathbb{E}_{d}\left[d_{k 0}^{-\alpha_{0}}\right] \mathbb{E}_{d}\left[d_{k k}^{\alpha_{d}}\right],
\end{aligned}
$$

where $(a)$ is due to the fact that the channel for D2D communication and the interference channel to the BS are independent; $(b)$ follows from the independence of path loss 


$$
\begin{aligned}
& \mathbb{E}_{d}\left[d_{k k}^{\alpha_{d}}\right]=\int d_{k k}^{\alpha_{d}} f\left(d_{k k}\right) \mathrm{d} d_{k k} \\
& =-\frac{2 d_{k k}^{\alpha_{d}+2}\left(-{ }_{2} F_{1}\left(\frac{1}{2}, \frac{\alpha_{d}+3}{2} ; \frac{\alpha_{d}+5}{2} ; \frac{d_{k k}^{2}}{4 R^{2}}\right) d_{k k}+\left(\alpha_{d}+2\right){ }_{2} F_{1}\left(-\frac{1}{2}, \frac{\alpha_{d}+3}{2} ; \frac{\alpha_{d}+5}{2} ; \frac{d_{k k}^{2}}{4 R^{2}}\right) d_{k k}-2\left(\alpha_{d}+3\right) R \cos ^{-1}\left(\frac{d_{k k}}{2 R}\right)\right)}{\pi\left(\alpha_{d}+2\right)\left(\alpha_{d}+3\right) R^{3}}
\end{aligned}
$$

and fading gain; $(c)$ follows from the Jensen's inequality; and (d) follows since $\mathbb{E}_{h}\left[\left|h_{i j}\right|^{2}\right]=1$.

Defining $\beta \triangleq \mathbb{E}_{d}\left[d_{k 0}^{-\alpha_{0}}\right] \mathbb{E}_{d}\left[d_{k k}^{\alpha_{d}}\right]$, from the average interference constraint in (19) and the bound in (20), we obtain an upper bound on the number of active D2D users as

$$
L \triangleq \sum_{k=1}^{K} x_{k} \leq \frac{c_{d}}{c_{0}} \frac{I_{\mathrm{th}}}{p_{r} \beta} .
$$

The bound in (21) is function of $\beta$ which in turn relies on the distributions of the distance of the D2D transmitters to their receivers as well as to the BS. These two distributions are dependent on the cell topology. Here, we consider a circular cell which is a good approximation for both hexagonal and square cell. To calculate $\mathbb{E}_{d}\left[d_{k 0}^{-\alpha_{0}}\right]$, the distribution of the distance between a D2D user and the BS is needed. We assume that users are uniformly distrusted over the cell area with distance $d_{\min } \leq d_{k 0} \leq R$ from the $\mathrm{BS}$, where $R$ is the cell radius. The lower bound on the distance is enforced so that the path loss model is valid. Then, the probability density function (PDF) of $d_{k 0}$ is

$$
f\left(d_{k 0}\right)=\frac{2 d_{k 0}}{R^{2}},
$$

from which we have the PDF of the random variable $y=d_{k 0}^{-1}$ as [15]

$$
g(y)=\frac{1}{y^{2}} f\left(\frac{1}{y}\right) .
$$

We calculate

$$
\begin{aligned}
\mathbb{E}_{d}\left[d_{k 0}^{-\alpha_{0}}\right] & =\mathbb{E}_{y}\left[y^{\alpha_{0}}\right]=\int_{1 / R}^{1 / d_{\min }} y^{\alpha_{0}} g(y) \mathrm{d} y \\
& =\frac{2}{R^{2}\left(\alpha_{0}-2\right)}\left(d_{\min }^{-\left(\alpha_{0}-2\right)}-R^{-\left(\alpha_{0}-2\right)}\right),
\end{aligned}
$$

for $\alpha_{0}>2$.

The PDF of the distance between two D2D nodes in a circular cell can be derived using the disk line picking described in [16, Ch. 6] as

$$
f\left(d_{k k}\right)=\frac{4 d_{k k}}{\pi R^{2}} \cos ^{-1}\left(\frac{d_{k k}}{2 R}\right)-\frac{2 d_{k k}^{2}}{\pi R^{3}} \sqrt{1-\frac{d_{k k}^{2}}{4 R^{2}}},
$$

with $D_{\min } \leq d_{k k} \leq D_{\max }$. Therefore, we can obtain the term $\mathbb{E}_{d}\left[d_{k k}^{\alpha_{d}}\right]$ from (26) on top of the page, in which ${ }_{2} F_{1}(a, b ; c ; z)$ is the Gaussian hypergeometric function. The integral in (26) is calculated numerically in the range $\left[D_{\min }, D_{\max }\right]$. Due to the channel inversion policy, the maximum allowed distance $D_{\max }$ between a D2D transmitter and its receiver has noticeable impact on the interference at the BS.
The value of $\beta$ in (21) is therefore depends on the path loss exponents and the cell radius, and can be computed from (24) and (26). Then, we have

$$
L \leq \frac{c_{d}}{c_{0}} \frac{I_{\mathrm{th}}}{p_{r} \beta\left(R, \alpha_{0}, \alpha_{d}\right)} \triangleq L_{\mathrm{ub}} .
$$

In the upper bound, $I_{\text {th }}$ is determined by the CUE's QoS requirement. To see how $L_{\mathrm{ub}}$ changes in parameters $\alpha_{d}$, the path loss exponent between two devices, $\alpha_{0}$, the path loss exponent between $\mathrm{BS}$ and a device, and $R$, the cell radius, we plot the normalized $L_{\mathrm{ub}}$ for a fixed CUE. The left plot in Fig. 2 depicts the decreasing behavior of $L_{\mathrm{ub}}$ in $\alpha_{d}$ when $\alpha_{0}=3.67$ and $R=200$. The reason for this exponential decrease is that increasing $\alpha_{d}$ results in more loss between D2D transmitters and receivers and due to the channel inversion power control policy, D2D transmitters increase their transmit power, thus create more interference at the BS. The behavior of $L_{\mathrm{ub}}$ is inverse in $\alpha_{0}$ as shown in the middle plot of Fig. 2 for $\alpha_{d}=4$. Increasing $\alpha_{0}$ makes the channel between the D2D transmitter and the BS worse, which leads to less interference at the BS. Therefore, more D2D users can be active. If both path loss exponents are the same (i.e., $\alpha_{d}=\alpha_{0}$ ), we observe in the right plot of Fig. 2 that the number of active D2D users will decrease with a lower slope as the path loss exponents grow larger. This shows that the increase in the D2D transmit power due to the channel inversion policy which leads to additional interference at the BS is slightly compensated by the degradedness of the channel to the BS. Fig. 3 shows that more D2D communications are possible if the cell size is increased with a fixed CUE, since in larger cells the probability that D2D users are spatially separated from the CUE is higher.

Using the bound in (27), the BS can estimate the maximum number of D2D users that can be active simultaneously without requiring CSI knowledge of the D2D users. That is, it can randomly allow $L=\min \left\{\left\lfloor L_{\mathrm{ub}}\right\rfloor, K\right\}$ D2D users to transmit on the uplink resource of the CUE. D2D users employ channel inversion as their power control policy and due to their lack of CSI knowledge, each active D2D user selects a random value as their channel gain based on the convolution of path loss distribution and fading gain distribution which can be computed numerically. This approach is also beneficial when a new D2D pair is discovered and the BS wants to determine if this user can be scheduled in the resource of the cellular user. The trade-off on the performance is that with this approach only the average interference constraint can be guaranteed and not the instantaneous QoS for the CUE as well as D2D users. In the next section, we study the CUE's outage 

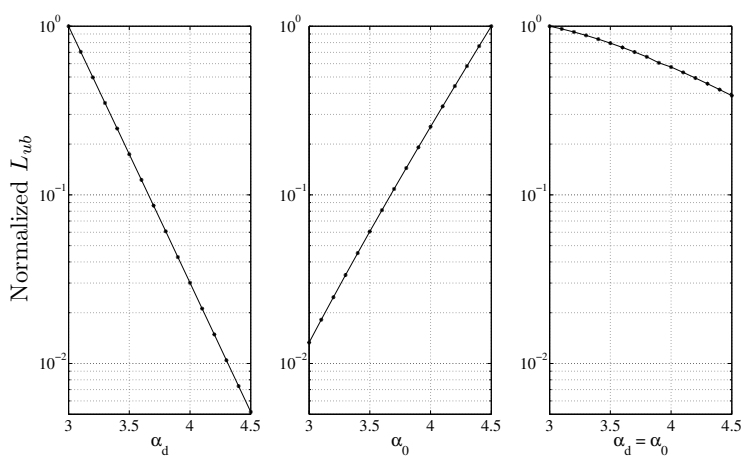

Fig. 2. Normalized $L_{\mathrm{ub}}$ vs. $\alpha_{d}$ (left), $\alpha_{0}$ (middle), and when $\alpha_{d}=\alpha_{0}$ (right).

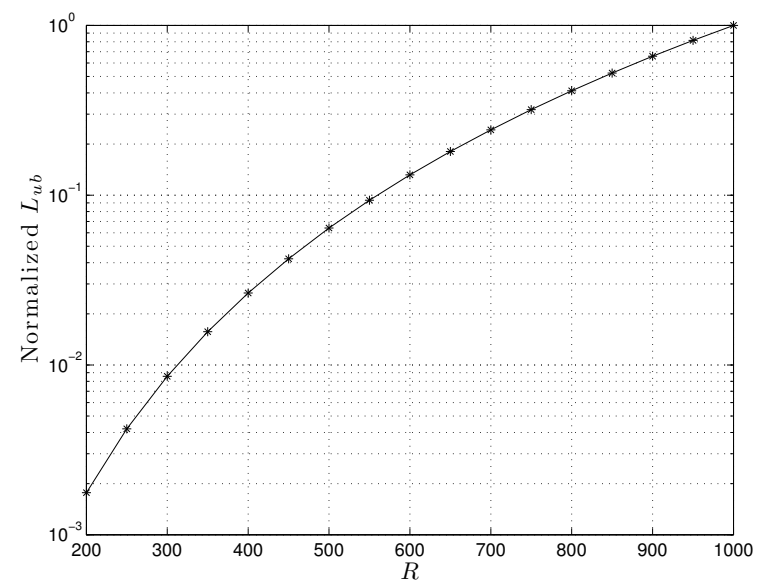

Fig. 3. Normalized $L_{\mathrm{ub}}$ as a function of $R$.

probability defined as

$$
P_{\text {out }}=\operatorname{Pr}\left(\sum_{k=1}^{K} x_{k} p_{k} G_{k 0}>I_{\mathrm{th}}\right),
$$

and show that the outage is actually very small.

\section{Performance Evaluation}

We evaluate the performance of our model under peak and average interference constraints by means of Monte-Carlo simulations. We consider a circular cell layout of radius $R$. The BS is located in the center of the cell. In each realization, one CUE and $K \mathrm{D} 2 \mathrm{D}$ transmitters are generated randomly with uniform distribution over the cell area with radius $d_{k 0} \in\left[d_{\min }, R\right]$ from the BS. D2D receivers are generated with distance $d_{k k} \in\left[D_{\min }, D_{\text {max }}\right]$ from their corresponding transmitters. We fix the transmit power of the CUE to $p^{c}=P_{\max }^{c}$. The channel model accounts for the effects of path loss and multi-path fading. The path-loss parameters are based on the non-lineof-sight (NLoS) scenario in [17]. The simulation parameters are given in Table I.

To solve the MILP optimization problem in (13)-(14), we used the GNU linear programming kit (GLPK) solver [18] which uses the branch-and-cut algorithm. We use this solution as a benchmark for our second approach in which no CSI from D2D users is needed. We use $K=8, \gamma_{0}^{\text {th }}=4 \mathrm{~dB}$, and $\gamma_{d}^{\text {th }}=2$ $\mathrm{dB}$ in the following simulation results unless otherwise stated.
TABLE I

SIMULATION PARAMETERS.

\begin{tabular}{lcc}
\hline Description & Parameter & Value \\
\hline Max. UE TX power & $P_{\max }^{c}, P_{\max }^{d}$ & $24 \mathrm{dBm}$ \\
Cell Radius & $R$ & $350 \mathrm{~m}$ \\
CUE SINR threshold & $\gamma_{0}^{\text {th }}$ & $4,8 \mathrm{~dB}$ \\
D2D SINR threshold & $\gamma_{d}^{\text {th }}$ & $2 \mathrm{~dB}$ \\
Noise power & $\sigma^{2}$ & $-107 \mathrm{dBm}$ \\
Carrier frequency & $f_{c}$ & $2 \mathrm{GHz}$ \\
System Bandwidth & $B$ & $5 \mathrm{MHz}$ \\
Max. D2D pair distance & $D_{\max }$ & $40 \mathrm{~m}$ \\
Min. D2D pair distance & $D_{\min }$ & $10 \mathrm{~m}$ \\
Min. device distance from BS & $d_{\min }$ & $10 \mathrm{~m}$ \\
Path loss exp. UE $\leftrightarrow \mathrm{UE}$ & $\alpha_{d}$ & 4 \\
Path loss exp. BS $\leftrightarrow \mathrm{UE}$ & $\alpha_{0}$ & 3.67 \\
Path loss coeff. UE $\leftrightarrow \mathrm{UE}$ & $c_{d}$ & $28.03 \mathrm{~dB}$ \\
Path loss coeff. BS $\leftrightarrow \mathrm{UE}$ & $c_{0}$ & $30.55 \mathrm{~dB}$ \\
Monte-Carlo simulation runs & MC & 10000 \\
\hline
\end{tabular}

Fig. 4 depicts the cumulative distribution function (CDF) of the cell sum rate in three approaches: conventional cellular transmission with no D2D communications, cellular transmission coexisting with multiple D2D users under peak interference constraint (PIC) where full CSI is known at the BS, and D2D communications under average interference constraint (AIC) in which no CSI is available. In our simulations, we assumed that if the CUE is in outage without any D2D communication, i.e., $I_{\text {th }}<0$, it does not transmit, and we removed these instances from the CDF plot. It can be observed that it is beneficial in terms of the cell sum rate that multiple D2D users share the spectrum with the CUE. Furthermore, the figure shows that our proposed approach based on AIC can still improve the system performance even though it does not require CSI knowledge from D2D users and the decision is based on randomness of the interference sources.

Fig. 5 shows the average number of active D2D users versus different number of available D2D users in the cell under PIC and AIC. As it can be seen, the number of active D2D users is higher in AIC case as this approach does not take into account the interference between D2D users and only guarantees the QoS for the CUE. In Fig. 6, the outage probability of the CUE at the BS caused by D2D transmissions under AIC is depicted. It is observed that the outage probability is low even though no signaling information is used in the decision process.

\section{CONClusions}

We studied the problem of spectrum sharing between a cellular user in the uplink and multiple D2D users under two different constraints: the peak and average interference constraints. In the former, we assumed that full CSI knowledge is available at the BS, based on which we obtain the optimal number of coexisting D2D communications while preserving certain QoS for both CUE and D2D users. In the later scenario, the locations of D2D users are assumed to be unknown and random along with their CSI. We find the upper bound on the number of D2D users that the system can accommodate based only on the average interference constraint. The proposed approach provides us with an important result that if a D2D pair requests to use the same resource of the cellular system, 


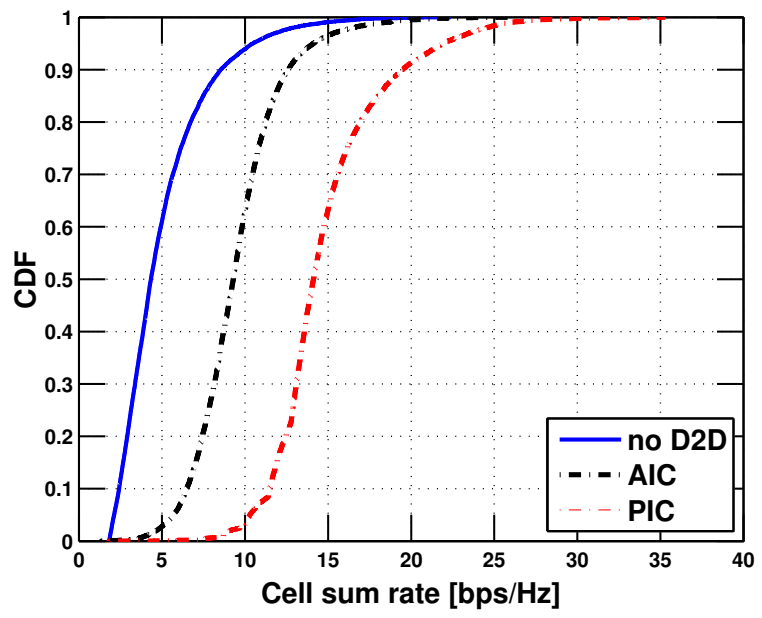

Fig. 4. CDF of the cell sum rate for different scenarios with $K=8, \gamma_{0}^{\text {th }}=4$ $\mathrm{dB}$, and $\gamma_{d}^{\text {th }}=2 \mathrm{~dB}$.

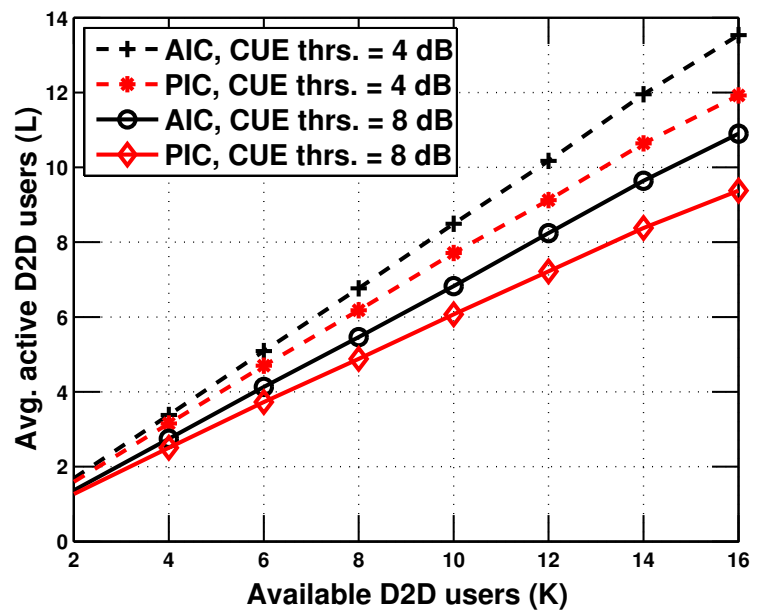

Fig. 5. Average (over $10000 \mathrm{MC}$ ) number of active D2D users $(L)$ vs. available D2D users $(K)$, with $\gamma_{0}^{\text {th }}=0,4 \mathrm{~dB}$ and $\gamma_{d}^{\text {th }}=2 \mathrm{~dB}$.

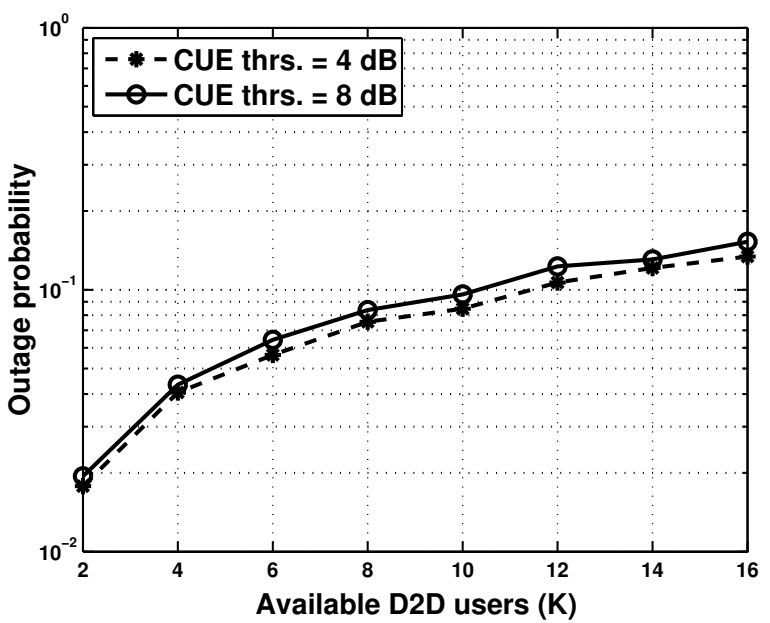

Fig. 6. CUE's outage probability vs. available D2D users $(K)$, with $\gamma_{0}^{\text {th }}=$ $0,4 \mathrm{~dB}$ and $\gamma_{d}^{\text {th }}=2 \mathrm{~dB}$. the BS, without any knowledge about the user's location, can decide if it can admit the user. We evaluated both approaches using Monte-Carlo simulations. Our results show a great potential for multiple coexisting D2D communications in the resource of one CUE. Moreover, we show that without imposing any signaling overhead in the decision process, it is possible to improve the system performance in terms of throughput while keeping the outage probability small.

\section{REFERENCES}

[1] L. Song, D. Niyato, Z. Han, and E. Hossain, Wireless Device-to-Device Communications and Networks. Cambridge University Press, to appear.

[2] L. Lei, Z. Zhong, C. Lin, and X. Shen, "Operator controlled deviceto-device communications in LTE-advanced networks," IEEE Wireless Commun. Mag., vol. 19, no. 3, pp. 96-104, Jun. 2012.

[3] G. Fodor, E. Dahlman, G. Mildh, S. Parkvall, N. Reider, G. Miklós, and Z. Turányi, "Design aspects of network assisted device-to-device communications," IEEE Commun. Mag., vol. 50, no. 3, pp. 170-177, Mar. 2012.

[4] K. Doppler, C. Yu, C. Ribeiro, and P. Jänis, "Mode selection for deviceto-device communication underlaying an LTE-advanced network," in Proc. IEEE Wireless Commun. Network. Conf. (WCNC), Sydney, Australia, Apr. 2010.

[5] S. Shalmashi, E. Björnson, S. B. Slimane, and M. Debbah, "Closedform optimality characterization of network-assisted device-to-device communications," in Proc. IEEE Wireless Commun. Network. Conf. (WCNC), Istanbul, Turkey, Apr. 2014.

[6] P. Jänis, C. Yu, K. Doppler, C. Ribeiro, C. Wijting, K. Hugl, O. Tirkkonen, and V. Koivunen, "Device-to-device communication underlaying cellular communications systems," IEEE Trans. Inf. Theory, vol. 2, no. 3 , pp. 169-178, Mar. 2009.

[7] C. H. Yu, O. Tirkkonen, K. Doppler, and C. B. Ribeiro, "On the performance of device-to-device underlay communication with simple power control," in Proc. IEEE Vehicular Technology Conf. (VTC), Barcelona, Spain, Apr. 2009.

[8] Y. Xu, R. Yin, T. Han, and G. Yu, "Interference-aware channel allocation for device-to-device communication underlaying cellular networks," in Proc. IEEE Int. Conf. on Commun. in China (ICCC), Beijing, China, Aug. 2012, pp. 422-427.

[9] C. Xu, L. Song, Z. Han, Q. Zhao, X. Wang, and B. Jiao, "Interferenceaware resource allocation for device-to-device communications as an underlay using sequential second price auction," in Proc. IEEE Int. Conf. on Commun. (ICC), Ottawa, Canada, Jun. 2012, pp. 425-429.

[10] G. Fodor and N. Reider, "A distributed power control scheme for cellular network assisted d2d communications," in Proc. IEEE Global Telecommun. Conf. (GLOBECOM), Houston, TX, Dec. 2011.

[11] S. Shalmashi, G. Miao, and S. B. Slimane, "Interference management for multiple device-to-device communications underlaying cellular networks," in Proc. IEEE Int. Symp. on Personal, Indoor, Mobile Radio Commun. (PIMRC), London, UK, Sep. 2013.

[12] E. Karipidis, E. Larsson, and K. Holmberg, "Optimal scheduling and QoS power control for cognitive underlay networks," in Proc. IEEE Int. Workshop on Computational Advances in Multi-Sensor Adaptive Process. (CAMSAP), Aruba, Dutch Antilles, Dec. 2009, pp. 408-411.

[13] A. Goldsmith, Wireless Communications. Cambridge University Press, 2005.

[14] S. Boyd and L. Vandenberghe, Convex Optimization. Cambridge University Press, 2004.

[15] A. Papoulis and S. U. Pillai, Probability, Random Variables and Stochastic Processes. McGraw-Hill, 2002.

[16] H. Solomon, Geometric Probability. Society for Industrial and Applied Mathematics, 1978.

[17] H. Xing and S. Hakola, "The investigation of power control schemes for a device-to-device communication integrated into OFDMA cellular system," in Proc. IEEE Int. Symp. on Personal, Indoor, Mobile Radio Commun. (PIMRC), Istanbul, Turkey, Sep. 2010, pp. 1775-1780.

[18] "GNU Linear Programming Kit (GLPK)," http://www.gnu.org/software/ glpk/. 